\title{
FreiwILlig AKTIV für GEFLÜCHTETE - GeflüChtete aktiv als Freiwillige. Wenn Menschen SICH für Menschen engagieren
}

Doris MoraVec, Volkshilfe Wien gemeinnützige Betriebs-GmbH, Wien

\section{INHALT}

1 Von Flucht, Migration und Vorurteilen.................................................................229

2 Freiwilligenarbeit im NGO-Kontext der Volkshilfe Wien .......................................2. 297

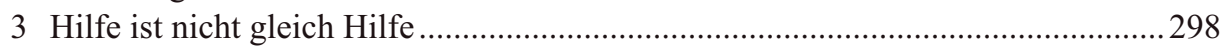

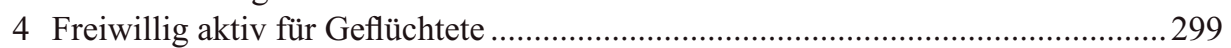

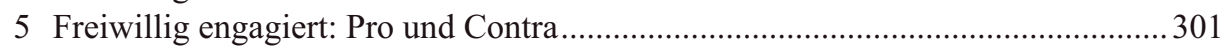

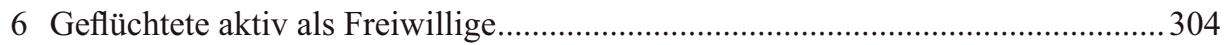

6.1 Exkurs: Rechtliche Rahmenbedingungen für Geflüchtete in Wien...................305

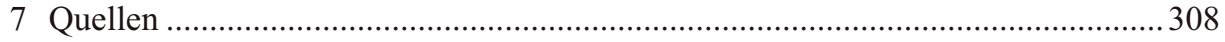

\section{Von Flucht, Migration und Vorurteilen}

„Ich wollte immer lernen. Aber als ich 16 Jahre war, mein Vater hat mir gesagt , du musst heiraten'. Dann war ich nur zu Hause, mit den Kindern. Meine Familie. Nicht lernen".

„Scheiße!", rutscht mir unkontrolliert heraus.

Sie lacht.

„Ja! Scheiße! Genau!“, erwidert sie, vehement nickend und mich anlächelnd. Sie wirkt erleichtert.

Als Rana ${ }^{1}$ zu mir ins Büro kommt, um sich wegen einer freiwilligen Tätigkeit zu informieren, erwische ich mich bei einer Reihe von Unterstellungen. Bis dato waren wir

Alle Namen in diesem Artikel wurden zum Schutz der Personen geändert. 
nur per E-Mail in Kontakt und ihr Deutsch wirkte ganz gut. Als ich die Tür zu meinem Büro öffne um sie hereinzubitten, steht sie mit ihrer 18-jährigen Tochter im Vorraum. Mein erster Gedanke: „Wieder jemand, die eine private Übersetzerin braucht“.

Doch wie so oft im Leben, bewahrheitet sich die Vermutung - das Vorurteil nicht: Ihre Tochter hatte sie bloß begleitet um sicher zu gehen, dass Rana die Adresse wirklich findet. Bevor wir ins Büro gehen verabschieden sie sich voneinander.

Unser Gespräch beginnt wie viele Gespräche mit geflüchteten Personen, die sich um eine freiwillige Tätigkeit bemühen: Auf meinen Einstieg „Erzähl mir ein bisschen von dir. Warum möchtest du bei uns aktiv werden?“, wird mit auswendig gelernten Sätzen in nahezu perfektem Deutsch geantwortet.

Und es geht weiter mit meinen Hypothesen, denn Rana ist nicht geflüchtet, wovon ich zunächst ausgegangen war. Nach einer kurzen Weile überwindet sie ihre anfängliche Schüchternheit und beginnt zu erzählen: Sie kommt aus Jordanien, hat fünf Kinder, allesamt mittlerweile erwachsen. Ihr ganzer Stolz, das merkt man. Sie sind alle sehr gebildet, studieren entweder aktuell oder haben, beziehungsweise werden bald studieren. Dann erzählt sie, dass sie auch immer lernen wollte. Doch mit 16 Jahren wurde sie mit ihrem Cousin verheiratet. Jetzt, da sie in Österreich sei, könne sie endlich lernen, sagt sie. Sie dürfe aber noch nicht arbeiten, weil ihre Aufenthaltsberechtigung an das Visum ihres Ehemanns gebunden sei. Demnächst wolle sie aber die Rot-Weiß-Rot-Karte Plus beantragen und eine Arbeit suchen. Ihren Mann müsse sie davon noch überzeugen, fügt sie mit einem Schmunzeln hinzu.

Rana ist 52 Jahre alt und trägt Kopftuch.

Ich denke im Anschluss noch viel über dieses Kennenlernen nach. Über die Vorurteile, die ich hatte. Über die Art, wie Rana sich nach ihrer anfänglich vorsichtigen und schüchternen Haltung allmählich zu öffnen begann und ehrlich sprach. Über die Tatsache, dass sie Kopftuch trägt und sie auf Grund dessen wahrscheinlich oft von Menschen falsch eingeschätzt, wenn nicht sogar unterschätzt wird. Und darüber, was eigentlich eine/n Geflüchtete/n ausmacht.

Es mag wie eine Themenverfehlung wirken, diesen Artikel mit der Geschichte einer Migrantin zu beginnen, wenngleich diese Publikation sich der Thematik Flucht widmet. Schließlich hatte Rana das Glück, mit Hilfe eines offiziellen Visums über ihren Ehemann nach Österreich zu kommen.

„Jordanien ist ein schönes Land. Aber nicht gut für Frauen“, sagt sie. Sie wolle nicht mehr zurück, fährt sie fort. - Auch Rana ist also geflüchtet. Nicht mit Schleppern. Nicht auf einem Boot. Nicht um ihr Überleben bangend. Aber geflüchtet ist sie. Vor Unterdrückung. Vor ihrem Land. 


\section{Freiwilligenarbeit im NGO-Kontext der Volkshilfe Wien}

Die Arbeit mit Freiwilligen und Geflüchteten begann für mich im Winter 2015/2016 in einem der Notquartiere für Geflüchtete der Volkshilfe Wien. Bis dahin hatte ich keine professionelle Erfahrung in diesem Bereich, konnte allerdings auf Kompetenzen aus 20 Jahren freiwilligem Engagement in einem Verein und ein Studium der Kultur- und Sozialanthropologie, welches sich unter anderem besonders mit den Themen Migration und Flucht auseinandersetzte, verweisen.

Mitte 2016 wurde ich mit der Projektleitung eines Patenschaftsprojekts für unbegleitete minderjährige Geflüchtete der Volkshilfe beauftragt und mit Januar 2017 übernahm ich die Abteilungsleitung des Freiwilligenmanagements der Volkshilfe Wien.

Die Volkshilfe ist eine gemeinnützige, konfessions- und parteiunabhängige Organisation, welche seit über 70 Jahren besteht und die soziale Landschaft in allen Bundesländern Österreichs mitgestaltet. Die Volkshilfe Wien ist unter anderem in den Bereichen Pflege und Betreuung, Delogierungsprävention, Arbeitsintegration, Betreuung von Geflüchteten sowie Kinder- und Jugendbetreuung tätig. Mit dem Freiwilligenmanagement wurde eine Stabsstelle geschaffen, welche den Koordinator/inn/en der Freiwilligenarbeit in den einzelnen Abteilungen unterstützend zur Seite steht und gleichzeitig den Freiwilligen eine professionelle Begleitung ihres Engagements bietet. In der Volkshilfe Wien sind knapp 500 Freiwillige und Ehrenamtliche sowie 1.700 Mitarbeiter/innen aktiv.

Eine unserer Aufgaben im Freiwilligenmanagement ist es, Erstgespräche mit Menschen zu führen, die sich für ein freiwilliges Engagement bei uns interessieren, aber noch nicht genau wissen, wie und wo sie sich einbringen möchten. Viele unserer Freiwilligen sind in der Unterstützung von Geflüchteten tätig, helfen beim Spracherwerb, beim Manövrieren durch den „Behördendschungel“ und beim allgemeinen „Ankommen“ in Österreich. Aber auch in der stationären Wohnungslosenhilfe, den sozialpädagogischen Wohngemeinschaften für Kinder und Jugendliche und diversen Projekten, sind viele engagierte Personen freiwillig aktiv.

Österreicher/innen, Studierende aus dem In- und Ausland, Migrant/inn/en aller Generationen und auch Geflüchtete finden uns im Internet über Bekannte, Freunde, oder Betreuer/innen. Oftmals wissen sie vor dem persönlichen Erstgespräch nicht viel über unsere Arbeit. Dies gibt uns die Gelegenheit längere Gespräche mit ihnen zu führen, ihnen von uns zu erzählen und sie von sich erzählen zu lassen. 


\section{Hilfe ist nicht gleich Hilfe}

Bei einem Erstgespräch mit einem/r potentiellen Freiwilligen ist es wichtig, die Motivation und Erwartungshaltung der Person zu eruieren. Zusätzlich ist es uns ein Bedürfnis bei dieser Gelegenheit herauszufinden, wie viel er/sie sich bereits mit dem Tätigkeitsfeld und/oder der Zielgruppe auseinandergesetzt hat. So können verfälschte Bilder und Vorurteile angesprochen, aber auch unrealistische Erwartungen thematisiert werden.

Freiwillige, die sich in der Flüchtlingshilfe engagieren, tun dies sowohl aus altruistischen als auch aus eigennützigen Motiven (SIMSA et al. 2016, p. 22; SCHWEINSCHWALlER 2018, p. 3). Nicht selten vermischen sich in der Praxis die unterschiedlichen Motivationen, ohne dass man dies bewusst wahrnimmt. Der Satz „ich möchte Menschen helfen“ ist dafür repräsentativ und kann in einem Erstgespräch als „Helfer/innen-Syndrom“ (SchmidBauer 1977) interpretiert werden, wenngleich das natürlich nicht immer den Tatsachen entspricht. Sich mit der eigenen Rolle als Freiwillige/r - als Helfer/in - auseinanderzusetzen, ist und bleibt allerdings wichtig. Sowohl für das eigene Wohl als auch für das Wohl des/der Klient/e/i/n und das Verhältnis der beiden zueinander.

„Helpers must be aware of their own emotional makeup and must be prepared to recognize that certain kinds of helper/client relationship may not be possible" (SCHEIN 2009, p. 45).

Im professionellen Kontext wird heute nicht gerne das Wort Hilfe verwendet. $\mathrm{Zu}$ sehr ist es mit einer Machtposition des/der Helfer/s/in über den/die Klient/e/i/n assoziiert. Zu sehr kann es eine bevormundende Haltung implizieren. Begriffe wie Begleitung oder Unterstützung werden daher heute bevorzugt (SCHWEINSCHWALLER 2018, p. 2).

Besonders in der Freiwilligenarbeit kann die Wortwahl hier sehr entscheidend sein: Jemanden zu begleiten, zu unterstützen, kann den Druck von dem/der Freiwilligen nehmen. Es wird als niederschwelliger wahrgenommen, als jemandem zu helfen. Zusätzlich kann es dabei unterstützen, die ungleiche Beziehung zwischen Helfer/in und Rezipient/e/i/n möglichst auf Augenhöhe zu halten.

In seiner Publikation Help. How to Offer, Give and Receive Help differenziert Edgar H. ScHEIN, Sozialpsychologe und -wissenschaftler, zwischen formeller, semi-formeller und informeller Hilfe. Er macht dabei keinen qualitativen Unterschied zwischen ihnen, sondern hebt sämtliche Formen von Hilfe als wichtig hervor und hält fest, dass diese voneinander lernen können und sollten (SCHEIN 2009). Darüber hinaus beschreibt er die allen Formen von Hilfe inhärente Ausgangssituation zwischen Helfendem $/ \mathrm{r}$ und Rezipient/e/i/n als ,,intrinsically unbalanced and role-ambiguous“ (ebenda, p. 31):

„Emotionally and socially, when you ask for help you are putting yourself “one down". It is a temporary loss of status and self-esteem not to know what to do next or to be unable to do it " (ebenda, p. $31 \mathrm{f}$.). 
Laut ScHEIN fühlt sich also der/die Klient/in oft unwohl, vielleicht sogar ängstlich oder gar schwach, wenn er/sie um Hilfe fragen muss oder sollte. Auf Seiten des/ der Helfer/s/in kann die neue Rolle einerseits ermächtigend wirken, allerdings auch zu Belastungen führen. Denn was tun, wenn das entgegengebrachte Vertrauen nicht ausreichend befriedigt werden kann? ScHEIN stellt fest, dass es Helfer/inne/n schwer fällt in dieser Situation zuzugeben, an ihre Grenzen gestoßen zu sein und nicht weiter zu wissen: „It is psychologically hard to give up such granted power, to say with humility, "I don’t know if I can help you or not" or "I cannot really help you"“ (ebenda, p. 34).

Auch ich machte zum Teil Erfahrungen dieser Art sowohl mit Freiwilligen als auch Mitarbeiter/inne/n, die in der Unterstützung von Geflüchteten tätig waren oder sind. Aus den besten Intentionen heraus kann es zur Weitergabe von falschen oder unvollständigen Informationen kommen, welche schnell eine Kettenreaktion auslösen. Und dies teilweise nur deshalb, weil man nicht gerne zugeben wollte, nicht helfen zu können. Es scheint dies eine zutiefst menschliche Reaktion zu sein.

Im Winter 2015/2016 arbeitete ich zunächst freiwillig in unterschiedlichen und anschließend Teilzeit in einem der Notquartiere für Geflüchtete in Wien. Auch ich fand mich in Situationen wieder, in welchen ich von Klient/inn/en um Hilfe gebeten wurde und sofort in eine Reaktion trat statt in eine Aktion. Vor der Versuchung, Menschen helfen zu können, d.h., einen tatsächlichen Einfluss haben zu können, waren auch meine Kolleg/inn/en und ich nicht immer gefeit.

Dies zeigt, dass sowohl Mitarbeiter/innen als auch Freiwillige in der Unterstützung von Personen stets Gefahr laufen können, dieser ,strong seductive emotional force operating to take advantage of this position“ zu unterliegen (SCHEIN 2009, p. 40).

\section{Freiwillig aktiv für Geflüchtete}

Das Jahr 2015 ist vielen Menschen in Österreich noch deutlich in Erinnerung. Egal ob aktiv in der Flüchtlingshilfe tätig oder passiv vor dem Fernseher verbringend, an kaum jemandem ging es spurlos vorüber. In dieser Zeit und auch danach war immer wieder die Rede von Ersthelfer/inne/n, ein Begriff, der bis dahin nahezu ausschließlich im Zusammenhang mit Erster Hilfe bei Unfällen verwendet wurde. ${ }^{2}$ Im Hinblick auf die rasant zunehmenden Bewegungen von Geflüchteten nach Europa im Jahr 2015 konnte dieser Begriff um zwei zusätzliche Bedeutungen ergänzt werden:

Einerseits wurde von haupt- und ehrenamtlichen Personen eine Art Erste Hilfe an Bahnhöfen, in Transit- und Notquartieren geleistet. Lebensmittel, Kleidung und andere Spenden mussten organisiert und verteilt, Schlafplätze zugewiesen und auch medizinische Erstversorgung geleistet werden.

Siehe Duden, <https://www.duden.de> 
Andererseits bewirkten die zunehmenden Fluchtbewegungen, dass viele Menschen sich zum ersten Mal als Freiwillige engagierten. Laut einer repräsentativen Untersuchung in Deutschland 2017 unterstützten seit 201555 Prozent der Bevölkerung Geflüchtete auf irgendeine Weise. 25 Prozent davon sind oder waren aktiv in der Flüchtlingshilfe tätig, wobei jede/r Dritte davon sich zum ersten Mal als Freiwillige/r engagierte. Für Deutschland bedeutet dies, dass neun Prozent der Gesamtbevölkerung im Rahmen der zunehmenden Fluchtbewegungen in und durch das Land im Jahr 2015, „zum ersten Mal zu aktiver Hilfeleistung motiviert“ (BMFSFJ 2017, p. 13) wurden.

In Österreich waren laut einer repräsentativen Bevölkerungsbefragung 2016 im Jahr 2015 ,rund zwei Prozent der Bevölkerung [in der Flüchtlingshilfe freiwillig] tätig“ (BMASK 2016, p. 7).

Ohne das zivilgesellschaftliche Engagement und das rasche Reagieren und Agieren dieser Helfer/innen wäre die damalige Situation in Österreich nicht zu bewältigen gewesen. Zusätzlich mussten österreichweit Abgrenzungen von Jobprofilen und Arbeitsbereichen vieler Mitarbeiter/innen situativ an die Praxis angepasst und neue Tätigkeitsfelder geschaffen werden. Es war eine herausfordernde Zeit. Für Organisationen, Fachkräfte, Freiwillige, Ehrenamtliche, aber natürlich vor allem auch für die Geflüchteten selbst.

Die Erfahrungen in und seit dem Sommer bzw. Herbst 2015 haben gemeinnützigen Organisationen - aber auch Freiwilligen - einiges gelehrt. Eine der Konsequenzen war daher die zunehmende Professionalisierung der Begleitung von Freiwilligen in diesem Bereich. In Wien wurden Schulungen und Fortbildungen zu den Thematiken Flucht, Trauma, Abgrenzung, aber auch zu den Herkunftsländern zum Beispiel von der MA 17, Magistratsabteilung für Integration und Diversität, und der Initiative POWER 2 HELP kostenfrei, beziehungsweise kostengünstig, für Freiwillige und Multiplikator/ inn/en angeboten. Auch Unterstützungsmechanismen wie Supervisionsgruppen und Reflexionstreffen wurden in vielen Organisationen, so auch in der Volkshilfe Wien, für Freiwillige in der Flüchtlingshilfe und Integrationsarbeit etabliert.

Seit 2015 veränderte sich das Feld der Flüchtlingshilfe und mit ihm die Ansprüche und Bedürfnisse von Klient/inn/en und Organisationen, aber auch die Tätigkeitsfelder von Freiwilligen. Von den ,neu definierten“ Ersthelfer/inne/n wechselten einige in ein kontinuierliches Engagement. Andere zogen sich aus der aktiven Unterstützung zurück. Die Gründe dafür sind unterschiedlich.

Zum einen entwickelten sich die Aufgabenbereiche für manche in eine Richtung, die ihnen nicht lag oder keine Freude bereitete. Schließlich ist zum Beispiel die punktuelle Ausgabe von Spenden oder Speisen eine ganz andere Tätigkeit als die kontinuierliche Lernbegleitung einer Person, die auf den Abschluss der Pflichtschule hinarbeitet. Aber nicht nur aufgrund sich verändernder Tätigkeitsfelder entschieden sich manche Freiwillige gegen eine Weiterführung ihres Engagements. Wurden sie 2015 noch als Held/inn/ en und Vorbilder der zivilgesellschaftlichen Größe Österreichs gefeiert, wandte sich die Aufmerksamkeit der Medien und Politik bald gegen die sogenannten „Gutmenschen“ (Simsa 2016, p. 65). Plötzlich mussten sich Menschen, die sich für Menschen enga- 
gierten, dafür rechtfertigen. Die Asylkoordination Österreich bietet im Zusammenhang damit ein „Argumentationstraining gegen Stammtischparolen“ an, welches vor allem auch von Freiwilligen wahrgenommen wird. ${ }^{3}$

Ein Engagement für Geflüchtete, so erleben wir es auch in unserem Patenschaftsprojekt für unbegleitete minderjährige Geflüchtete Buddies for Refugees, ist heute mehr denn je ein politisches Statement. Seit Projektstart im Mai 2016 wurde die Aufgabe, neue Buddies (Pat/inn/en) für das Projekt zu finden, immer arbeitsintensiver und herausfordernder. Auch andere Patenschaftsprojekte in Wien und den Bundesländern erzählen in den jährlichen Vernetzungstreffen von den gleichen Entwicklungen. Jene, die sich aber weiterhin engagieren, tun dies trotz der aktuellen Stimmung gegen sie und die Geflüchteten. Dabei braucht es eine „dicke Haut“, vor allem im Umgang mit Behörden, wie uns einer der Buddies erzählte. Er und sein Partner teilten sich die Aufgabe der Patenschaft und wechselten sich bei Terminbegleitungen ab. Sie und auch wir schrieben Briefe darüber, wie gut sich Mohamad entwickelte, wie sehr sich sein Deutsch verbesserte und über seine hohe Motivation, in Österreich eine Ausbildung zum Kindergartenpädagogen zu machen. Sie inkludierten Mohamad in ihren Freundeskreis, unternahmen Ausflüge und tauschten sich über die unterschiedlichen Kuriositäten ihrer Heimatländer aus. Wie viele andere unserer Freiwilligen betonten auch sie die Reziprozität in ihrer Beziehung zu Mohamad. Sie erlebten ihre Freiwilligenarbeit nicht als einseitige Hilfe ihrerseits für den geflüchteten Burschen, sondern als einen Austausch.

Anfang Dezember 2018 kehrte Mohamad, nachdem er den zweiten negativen Asylbescheid erhalten hatte und ab diesem Zeitpunkt in ständiger Angst um eine Abschiebung lebte, freiwillig nach Afghanistan zurück. Der Druck dieser Situation war zu viel für ihn geworden. Die „Freiwilligkeit“ ist für uns dabei in Frage zu stellen.

Seine Buddies erzählten später, dass sie sich zwar noch in einem Schockzustand befänden, es aber nicht bereuten, Mohamad für zwei Jahre so intensiv in ihren Alltag inkludiert gehabt zu haben. Einzig die wiederholte, respektlose und oftmals schlicht erniedrigende und unprofessionelle Art der Beamt/inn/en im Asylverfahren habe ihnen immer wieder massive Dämpfer in ihrer Motivation verpasst. Rückmeldungen wie diese gibt es Tausende. Eine Besserung scheint nicht in Sicht. Ein Grund mehr, Freiwillige in diesem Bereich professionell vorzubereiten und zu unterstützen.

\section{Freiwillig engagiert: Pro und Contra}

Unabhängig von den Beweggründen, warum Menschen in ein für sie unbekanntes Land ziehen: Lokale Kontakte sind von Anfang an von unschätzbarem Wert. Wir bezeichnen unsere Freiwilligen in diesem Zusammenhang gerne auch als „Botschafter/ innen Österreichs“, weil sie oft der erste Kontakt auf persönlicher Ebene in dem neuen Land sind und wesentlich zum Verstehen desselben beitragen können.

Details dazu unter „Schule \& Vorträge“ auf $<$ www.asyl.at $>$ 
Han-Broich beschreibt Integration als einen Prozess, in welchem einander Migrant/inn/en und die Aufnahmegesellschaft wechselseitig annehmen „und einem „größeren Ganzen“ anpassen“ (2012, p. 122). Darüber hinaus differenziert sie drei Unterkategorien, welche, so ein Gleichgewicht vorhanden ist, positiv wirken können: Die seelisch-emotionale Integration, die kognitiv-kulturelle Integration und die sozial-strukturelle Integration (2012, p. 122 f.). Die „soziale Integration“ als Teil der dritten Unterkategorie beschreibt HAN-BROICH dabei als „Angleichung in den Beziehungsmustern in Form interethnischer Kontakte und institutioneller Partizipation (Freund- und Partnerschaften, Vereinsmitgliedschaften)“(2012, p. 123). Anders ausgedrückt könnte man diese interethnischen Kontakte und die institutionelle Partizipation auch als soziales Kapital nach BouRdIEU, also als „Ressourcen, die auf der Zugehörigkeit zu einer Gruppe beruhen“, bezeichnen (1983, p. 190 f., Hervorh. i. Original). Im Alltag von Geflüchteten stellen Freiwillige oftmals den ersten Kontakt dieser Art dar. Wenngleich die professionelle Begleitung und Unterstützung von Fachkräften niemals ersetzt werden kann und von unschätzbarem Wert für die Betroffenen ist, nimmt die freiwillige Begleitung bei Geflüchteten einen anderen, teilweise durchaus als höherwertig empfundenen, Stellenwert ein.

Folgende Aussage eines Geflüchteten verdeutlicht dies eindrucksvoll:

„(..) dass diese Menschen, und damit sind auch nicht nur die Ehrenamtlichen gemeint, sondern auch überhaupt die Menschen, die positiv gegenüber den nicht Deutsch sprechenden Leuten gestimmt sind, dass die in dieser Dunkelheit so kleine Leuchten oder auch Wegweiser sind. Wenn diese Leuchten in dieser (völligen) Dunkelheit nicht da wären, dann könnte man das Leben abschreiben und aufgeben " (HAN-BROICH 2012, p. 136).

Auf Seiten der freiwillig engagierten Lokalbevölkerung kommen, wie bereits erwähnt, verschiedene Motivationen sowohl eigennütziger als auch altruistischer Natur zum Tragen. Dass es für Freiwillige zunehmend wichtiger wird, am Geschehen teilzuhaben, eigene Potenziale und Kompetenzen einzubringen und weiterentwickeln zu können, wird dabei heute immer deutlicher (SAMOchowIEC et al. 2018).

In einer Publikation des NPO \& SE Kompetenzzentrums der Wirtschaftsuniversität Wien formulierte dies ein Freiwilliger folgendermaßen: „Ich hatte das Gefühl, ich verpass` da was. Es war auch durchaus dabei, dass ich Menschen helfen wollte. Aber mein Selbstbild des Menschen, der da hilft, war herausgefordert. Weil ich nichts gemacht habe und alle anderen schon. Ich wollte da irgendwie mitmachen und ein Teil des Ganzen sein“" (SimsA et al. 2016, p. 23).

Gemäß meiner Erfahrung trifft diese Mischung von selbstlosen und eigennützigen Motiven auch auf andere soziale Tätigkeitsfelder von Freiwilligen, nicht nur auf die Flüchtlingshilfe, zu. Helfen wird somit zu etwas Erstrebenswertem, nicht nur um des Helfens willen, sondern auch um die eigene Persönlichkeit und mitunter den eigenen Lebenslauf weiterzuentwickeln. Es muss dies nicht als verwerflich angesehen werden, 
sondern scheint eher ein Spiegel unserer Zeit zu sein. Internationale Freiwilligendienste wie jene organisiert von Grenzenlos - Interkultureller Austausch, Service Civil International (SCI), Habitat for Humanity, Voluntary Service Overseas (VSO) und viele andere, erleben wahrscheinlich auch aufgrund dessen in den letzten Jahren einen so großen Aufschwung. Die 1970 gegründete International Association for Volunteer Effort (IAVE) veranstaltet alle zwei Jahre eine Freiwilligen-Konferenz für Organisationen, die mitunter oder hauptsächlich internationale Freiwilligendienste vermitteln. 2018 fand diese Konferenz mit 726 Teilnehmer/inne/n aus 78 Ländern in Augsburg, Bayern, statt. ${ }^{4} \mathrm{Jede} / \mathrm{r}$ dritte Augsburger/in sei freiwillig tätig, erzählte der Oberbürgermeister stolz bei seiner Eröffnungsrede. Und das nicht allein seit, aber besonders während der herausfordernden Fluchtbewegungen von 2015/2016. Hier scheint sich also Freiwilligenarbeit sogar als Teil des Leitbilds der Stadt etabliert zu haben.

Außen vor blieb bei dieser Tagung jedoch die kritische Auseinandersetzung mit den mitunter problematischen Facetten von Freiwilligenarbeit: Das (leider manchmal durchaus begründete) Misstrauen von Mitarbeiter/inne/n, ob unbezahlte Freiwillige ihre bezahlte Stelle ersetzen, die Tatsache, dass Freiwillige leider immer wieder für Hilfstätigkeiten ausgenutzt werden, aber auch die Zunahme an Corporate-VolunteeringGruppen-Anfragen von großen Konzernen an Vereine, NGOs etc., welche für letztere mitunter mehr Aufwand als Mehrwert bedeuten. Allgemein schien der Fokus dieser Konferenz stark auf den Bedürfnissen von Freiwilligen und jenen, die Freiwillige koordinieren, zu liegen, wohingegen die Perspektive der Empfänger/innen der Hilfe, der Klient/inn/en, eher im Hintergrund zu verschwinden schien. Eine Perspektive, die gerade bei internationalen Freiwilligendiensten nicht außer Acht gelassen werden sollte:

Wie wirkt es sich auf eine Gesellschaft aus, wenn verhältnismäßig reiche junge Menschen des globalen Nordens im globalen Süden helfen? Was sind die Langzeiteffekte auf die lokale Bevölkerung, wenn nur wenige Auserwählte Gastgeber/innen (,host families“) für die internationalen Volunteers sein dürfen? Welche Auswirkungen hat es besonders auf kleine Kinder in den angebotenen Englischklassen, Nachmittagsbetreuungen, Waisenhäusern etc., wenn sie sich alle paar Wochen emotional auf eine neue Runde Freiwilliger einlassen müssen?

Mit der Zunahme von internationalen Freiwilligendiensten, aber auch auf nationaler Ebene sollte eine Auseinandersetzung mit dieser Seite der Freiwilligenarbeit heute selbstverständlich sein. Eine Möglichkeit dafür ist die Ermächtigung von Klient/inn/en aus der Rolle des/der Hilfeempfänger/s/in heraus, in jene des/der aktiv Helfenden zu schlüpfen und die damit ermöglichte Partizipation und Begegnung auf Augenhöhe zu erleben.

\footnotetext{
$4 \quad$ Mehr zur World Volunteer Conference 2018 kann auf der Website der International Association for Volunteer Effort (IAVE) unter $<$ www.iave.org $>$ nachgelesen werden.
} 


\section{Geflüchtete aktiv als Freiwillige}

Neben dem Engagement der einheimischen Zivilgesellschaft waren und sind auch geflüchtete Personen zunehmend als Freiwillige tätig. In unserer Arbeit im Freiwilligenmanagement führen wir zahlreiche Erstgespräche mit Menschen aus den verschiedensten Ländern, mit unterschiedlichen Deutschkenntnissen. Oft war ich dabei von ihnen sehr beeindruckt, wie im Fall von Rana, manchmal aber auch enttäuscht, wie bei einem jungen Mann der, ohne dass wir einander kannten, eine Woche vor seinem Asylinterview mit dem Anliegen zu mir kam, ich möge ihm eine Bestätigung über eine freiwillige Tätigkeit ausstellen, die er allerdings nie vollbracht hatte, einzig weil es für seinen Asylantrag hilfreich sein könnte.

Die Erfahrungen in den Gesprächen mit potentiellen Freiwilligen waren und sind sehr unterschiedlich. Dabei kommt es nicht darauf an, woher eine Person kommt oder wie lange sie bereits in Österreich ist. Oftmals kommen zum Beispiel Menschen zu mir, die selbst vor einigen Jahren nach Österreich geflüchtet sind, und aus eigener Erfahrung wissen, wie schwer besonders der Anfang hier sein kann. Sie erzählen mir von den sprachlichen Herausforderungen, von der Trennung der Familie auf oder vor der Flucht und vom langen Warten, bis sie wieder vereint werden konnten. Und sie erzählen mir von den vielen Menschen, die ihnen beim „Ankommen“ in Österreich geholfen haben: Betreuer/innen und Berater/innen, die über die Grenzen der Definition ihrer Tätigkeit hinaus denken, Menschen in der eigenen Community, die selbst einiges erlebten, bis sie sich hier auch nur ansatzweise zu Hause fühlen konnten, und natürlich von Freiwilligen. Männer und Frauen, die in ihre damaligen Grundversorgungsunterkünfte kamen und fragten, ob jemand Hilfe brauche. Teilweise entwickelten sich aus spontanen Hilfeleistungen Freundschaften, die bis heute andauern. Und als sie im Fernsehen von der „Flüchtlingswelle“ hörten, erzählen sie, da wussten sie, dass sie etwas davon weitergeben wollten.

Die Geschichten dieser Menschen sind durchwegs Erfolgsgeschichten. Sie erzählen mir, dass es anfangs nicht einfach war, aber dass sie Hilfe erfahren - und auch angenommen - haben. Sie sagen, dass nun auch sie Österreich und die Menschen mit ähnlichen Geschichten wie sie selbst unterstützen wollen. Den Satz „Ich habe schon lange überlegt etwas zu tun, und jetzt geht es sich in meinem Leben auch endlich aus“ höre ich oft, wenn ich in Erstgesprächen nach der Motivation für das freiwillige Engagement frage. Nicht nur von selbst geflüchteten oder migrierten Personen, sondern von Menschen aller „Hintergründe“. In der Freiwilligenarbeit schätzen wir diese Freiwilligen besonders, denn sie haben sich bereits etwas damit auseinandergesetzt, was es bedeutet sich freiwillig zu engagieren.

Einen wesentlichen Faktor in der Freiwilligenarbeit in unserer NGO bilden leider die Sprachkenntnisse. „Leider“, weil wir immer wieder Personen bei uns im Gespräch haben, die genau wegen dieser mangelnden Sprachkenntnisse gerne freiwillig tätig werden wollen: Um zu üben, um mit Menschen deutscher Muttersprache in Kontakt zu 
kommen. Wenn man in einem Grundversorgungshaus mit 70 bis 100 anderen Personen (manchmal auch mehr) lebt, von denen viele die gleiche(n) Sprache(n) sprechen (viele wachsen mehrsprachig auf), fehlt es oft an Möglichkeiten, die deutsche Sprache zu üben.

\subsection{Exkurs: Rechtliche Rahmenbedingungen für Geflüchtete in Wien}

Österreich ist verpflichtet, geflüchteten Personen ,während des Verfahrens zur Feststellung der Flüchtlingseigenschaft (Asylverfahren) eine angemessene Unterkunft und Betreuung bereitzustellen (EU-Aufnahmerichtlinie)“ (AsYLKOORDINATION 2016b). ${ }^{5}$ Seit 2004 definiert die Grundversorgungsvereinbarung diesbezüglich österreichweite Aufnahmebedingungen und Kostenhöchstsätze. ${ }^{6}$ Die Leistungen der Grundversorgung umfassen Unterkunft und Verpflegung, Krankenversicherung, Bekleidungshilfe, Schulbedarf für Schüler/innen, Information, Beratung und Betreuung, Fahrtkosten bei behördlichen Ladungen und Überstellungen sowie Angebote zur Tagesstruktur (AsYLKOORDINATION ÖSTERREICH 2016b, p. 2). Die dabei entstehenden Kosten werden zwischen Bund und Ländern in einem Schlüssel von 60:40 aufgeteilt (AsYLKOORDINATION ÖSTERREICH 2016b, p. 1; IOM 2014, p. 10).

Zusätzlich zu den vorgegebenen Rahmenbedingungen der Grundversorgung für ganz Österreich existieren individuelle Vereinbarungen der Bundesländer, welche im Bund-Länder-Koordinationsrat verhandelt werden. Darüber hinaus gibt es Bundesländerquoten, nach welchen jedes Bundesland entsprechend der Wohnbevölkerung Geflüchtete aufnehmen muss (AsylKoORdinAtion Österreich 2016b, p. 2). So kommt es, dass die Leistungen der Grundversorgung in der Praxis je nach Bundesland anders umgesetzt und dadurch Asylwerber/innen in unterschiedlichem Ausmaß begleitet und betreut werden. ${ }^{7}$

Die Vorgabe für den maximalen Betreuungsschlüssel „für Information, Beratung und soziale Betreuung (exkl. Dolmetscherkosten)" innerhalb der Grundversorgung in Österreich, ist 1:170. ${ }^{8}$ Laut Asylkoordination Österreich kommt im Schnitt ein/e

Die Bedingungen, nach welchen eine Person zum Asylverfahren zugelassen wird, basieren auf der Ratifizierung der Genfer Flüchtlingskonvention (GFK) sowie hinsichtlich der Verteilung in Europa auf der „Dublin Verordnung“, welcher Österreich 1997 nach dem Eintritt in die EU beitrat. Seit 2013 kommt sie in seiner dritten Überarbeitung als „Dublin-III-VO“ zur Anwendung. Eine Zusammenfassung dieser Richtlinien kann auf der Website der Asylkoordination Österreich $<$ www.asyl.at $>$ nachgelesen werden (AsYLKOORDINATION 2016a).

${ }^{6}$ Weiterführende Informationen zur Grundversorgungsvereinbarung nach Art. 15a B-VG (Bund - Länder) sind im Rechtsinformationssystem des Bundes (RIS) unter $<$ https://www.ris.bka. gv.at/> abrufbar.

Dieser Artikel bezieht sich auf Informationen der Asylkoordination Österreich und fokussiert sich auf die Bedingungen der Grundversorgung in Wien, nach welchen auch in der Volkshilfe Wien gearbeitet wird.

$8 \quad$ Siehe Fußnote 7. 
Sozialarbeiter/in, also eine qualifizierte Fachkraft, auf 140 Geflüchtete (AsYLKOoRdiNATION ÖSTERREICH 2016b, p. 3).

Die Verantwortungsbereiche und Qualifikationen von Mitarbeiter/inne/n in der Wiener Grundversorgung sind dabei vielfältig. Je nach Aufgabengebiet sind dies Sozialarbeiter/nnen, Sozialpädagog/inn/en, psychosoziale und Flüchtlingsberater/innen sowie Menschen mit wenig bis gar keiner fachspezifischen Ausbildung, die aber zum Beispiel die Muttersprache der Zielgruppe sprechen.

In Wien kommt in einer Unterkunft für geflüchtete Familien und/oder alleinstehende erwachsene Personen ein Betreuungsschlüssel von 1:55 zum Tragen. Das heißt, dass eine Vollzeitkraft auf 55 Geflüchtete innerhalb einer Grundversorgungseinrichtung entfällt. Die Aufgaben der Mitarbeiter/innen in diesen Quartieren reichen von der Erstellung von Putzplänen, über die Auszahlung von Verpflegungsgeld bis hin zu informellen Gesprächen mit den Bewohner/inne/n, um das Zusammenleben im Haus zu unterstützen. Belastungsfaktoren können dabei beispielsweise eine lange Verfahrensdauer oder das Zusammenleben auf engem Raum sein.

In einer organisierten Unterkunft dieser Art werden für die Unterbringung und Verpflegung pro Person und Tag maximal 21 Euro an den Unterkunftsgeber bezahlt (AsYLKOORDINATION ÖsTERREICH 2016b, p. 2). In Wien dürfen Grundversorgungs-unterbringungen nur von gemeinnützigen Trägern geführt werden, welche vom Fonds Soziales Wien (FSW) beauftragt werden. Es ist also nicht möglich, dass Gasthäuser, Hotels und dergleichen Unterkünfte für Geflüchtete in der Grundversorgung zur Verfügung stellen. Gemeinnützige Träger wie die Caritas, die Volkshilfe, der Arbeiter-SamariterBund und andere führen in Wien Grundversorgungshäuser als Teilversorgungsquartiere. Dies bedeutet, dass von den 21 Euro pro Tag und Person 5,50 Euro an die Klient/inn/en als Verpflegungsgeld zur Selbstversorgung ausbezahlt werden. Die verbleibenden 15,50 Euro werden für Miete, Stromkosten, Instandhaltung der Einrichtung, Personalkosten und Ähnliches verwendet.

Artikel 7 der Grundversorgungsvereinbarung beschreibt Sonderbestimmungen für unbegleitete minderjährige Geflüchtete, ${ }^{9}$ wobei auch diese in den Bundesländern unterschiedlich umgesetzt werden. In Wien werden sie zum großen Teil in Wohngemeinschaften/Wohnheimen mit einem Betreuungsschlüssel von 1:15 untergebracht. ${ }^{10}$

\footnotetext{
9 „Unbegleitete Minderjährige im Asylverfahren sind Fremde unter 18 Jahren, bei denen kein Elternteil oder Obsorgeberechtigter in Österreich anwesend ist. [Sie] werden in speziellen Unterkünften untergebracht und erhalten besondere Betreuung und Versorgung“ (Bundesministerium für Inneres (BM.I): <www.bmi.gv.at>. In der Praxis wird das negativ konnotierte Wort „Fremde“ zumeist mit „Flüchtlinge“ ersetzt, wobei auch dieses mittlerweile nicht mehr unumstritten ist. In diesem Artikel wird deshalb der Bezeichnung „Geflüchtete“ der Vorrang gegeben.

${ }_{10}$ Bei unbegleiteten minderjährigen Geflüchteten kommen zahlreiche Sonderregelungen zum Tragen, sowohl in der Grundversorgungsvereinbarung als auch in der Dublin-III-VO allgemein. So kann zum Beispiel die Obhut auch an (entfernte) Verwandte, welche ebenso in Österreich sind, übertragen oder eine Pflegschaft übernommen werden (Details dazu: siehe Website der Asylkoordination Österreich <www.asyl.at>). In diesem Artikel wird nur auf die am weitesten verbreitete Grundversorgungspraxis in Wien eingegangen.
} 
Dabei werden höchstens 15 Minderjährige pro Wohngemeinschaft mit einem Tagsatz zwischen 40,50 und 95 Euro pro Minderjährigem/r und Tag betreut. Im Vergleich: In Einrichtungen der Kinder- und Jugendhilfe beginnen Tagsätze pro Kind bzw. Jugendli$\mathrm{chem} / \mathrm{r}$ und Tag bei 120 Euro je nach Betreuungsbedarf (AsYlKoORDINATION ÖSTERREICH 2017, p. 3).

Der Rahmen der Betreuung Geflüchteter innerhalb der Grundversorgung ist in Wien folglich besser als lediglich den Mindestanforderungen entsprechend gestaltet. In der Praxis ist die tägliche Arbeit der Fachkräfte in der Flüchtlingsbetreuung und der Hausleiter/innen zu einem wesentlichen Teil von bürokratischen und administrativen Vorgaben des Fördergebers beeinflusst. Besonders in diesen Unterkünften können Freiwillige den Alltag der Klient/inn/en oft mit den einfachsten Mitteln schöner gestalten. Eine unserer Freiwilligen, die bereits seit September 2015 mehrmals pro Woche in einem unserer Grundversorgungshäuser für alleinstehende geflüchtete Männer tätig ist, formulierte dies in einem offiziellen Rahmen auf die Frage, ob sie nicht eine bezahlte Stelle ersetze, so: „Ich sehe mich nicht als Ersatz für bezahlte Arbeit. Ich sehe das, was ich tue, als Bonus, als ,add-on“. Und ich kann mir ja selbst aussuchen, wie viel oder wenig ich machen will. Das schreibt mir keiner vor".

„Neben Autonomie, also dem Gefühl, selbst entscheiden zu können, und den dafür benötigten Freiräumen ist die soziale Eingebundenheit der zweite wichtige Faktor für das Sinnpotenzial einer Aufgabe“ (SAmochowiEC et al.2018, p. 26). Dieser Wunsch nach Eingebundenheit, nach Teilhabe, nach Partizipation, spiegelt sich auch in dem Bemühen vieler Geflüchteter um ein freiwilliges Engagement wider.

Viele von ihnen wurden bereits kurz nach der eigenen Ankunft in Wien als Freiwillige aktiv. Mohamad, der zuvor erwähnte Geflüchtete, der als unbegleiteter Minderjähriger 2015 nach Wien kam und Ende 2018 ,freiwillig“ nach Afghanistan zurückkehrte, war bereits wenige Wochen nach seiner Ankunft in Wien selbst am Hauptbahnhof aktiv. Er half bei Übersetzungen von Dari auf Englisch mit und unterstützte bei der Verteilung von Essenspaketen an Ankommende. Er erzählte auch, dass es ihm besonders große Freude bereitete, den ankommenden Kindern ein Lächeln in ihre erschöpften Gesichter zu zaubern. Ob er seinen Traum von einer Karriere als Kindergartenpädagoge in Afghanistan wird erfüllen können, bleibt offen.

Eine Geschichte mit einem etwas anderen Verlauf ist jene von Faruq. Auch er kommt aus Afghanistan, flüchtete allerdings mit seiner Familie nach Österreich, und lebte als Asylwerber in einem der Grundversorgungshäuser der Volkshilfe Wien. Im Frühjahr 2017 kontaktierte uns sein Berater und erzählte uns, dass Faruq sehr gerne freiwillig tätig werden würde, vor allem auch um seine Sprachkenntnisse zu verbessern. Im Juni 2017 konnte er dann in der Sozialberatungsstelle der Volkshilfe Wien beginnen, wo er bis heute regelmäßig dabei hilft, Lebensmittelpakete an Menschen, die an der Armutsgrenze leben, zu verteilen. 2018 erhielten seine Familie und er subsidiären Schutz, und er begann intensiv nach einer bezahlten Arbeit zu suchen - als Freiwilliger blieb er allerdings weiter aktiv. Da es schwer für ihn ist, einen bezahlten 
Job zu finden, engagierte er sich zusätzlich für ein paar Wochen in einer der sozialpädagogischen Wohngemeinschaften der Volkshilfe Wien. Leidenschaftlicher Koch, der er ist, zauberte er mit guter Hausmannskost so manches Lächeln auf die Gesichter der dort untergebrachten afghanischen Jugendlichen, die sich nach Essen aus der Heimat gesehnt hatten.

Im Austausch mit anderen gemeinnützigen Organisationen in Wien wird wiederholt das Engagement von Geflüchteten in der Freiwilligenarbeit Thema. Eine der größten Herausforderungen dabei ist es, Menschen mit geringen Deutschkenntnissen in Tätigkeitsbereiche zu vermitteln, die sowohl für sie als auch für die Organisation und die Klient/inn/en einen Mehrwert aufweisen. Jemand, der/die sich auf Deutsch nur schlecht verständigen kann, wird zum Beispiel keine Lernhilfe geben können. Er/sie könnte allerdings als freiwillige/r Begleiter/in von älteren Personen eingesetzt werden, die eine zweite Person benötigen, um die Motivation zu finden, wieder aus dem Haus zu gehen. Ein gutes „matching“, also eine überlegte Vermittlung eines/r potentiellen Freiwilligen zu seinem/ihrem möglichen und passenden Tätigkeitsbereich, ist dabei einmal mehr entscheidend. Manchmal bedarf es diesbezüglich einer kreativen Lösung, auch müssen neue Wirkungsbereiche für Freiwillige erst geschaffen werden. Dies, wie auch die professionelle Gestaltung von Freiwilligenarbeit im Allgemeinen, braucht Ressourcen und Menschen, die über den Tellerrand hinaus blicken können.

Begegnungen mit Personen wie Rana bleiben mir lange im Gedächtnis - ihre Biografie, ihre offene Art, ihre Kraft und Selbstermächtigung sowie ihre starke Motivation etwas zu tun, zu lernen, zu partizipieren. Ranas Geschichte ist kein Einzelfall, das wissen Menschen, die sich, egal ob beruflich oder freiwillig, in der Integrationsarbeit engagieren. Wir kennen unzählige Geschichten von Menschen, die sich für Menschen engagieren wollen.

Das Engagement von Menschen, die selbst Notlagen erlebt haben und sich engagieren wollen um anderen beizustehen, zeigt uns die Solidarität von benachteiligten Personen. Diese Entwicklung stimmt uns hoffnungsvoll und weist einmal mehr auf die vielfältigen Potenziale von freiwilligem Engagement hin.

\section{Quellen}

AsylKoordination Österreich (2016a), Die Dublin-Verordnung. AsylKOORDINATEN. Infoblatt der Asylkoordination Österreich Nr. 5. Wien, asylkoordination österreich. Stand 2016.

AsYLKOORDINATION ÖsterReich (2016b), Grundversorgung. AsylKOORDINATEN. Infoblatt der Asylkoordination Österreich Nr. 2. Wien, asylkoordination österreich. Stand 2016.

AsylKoordination ÖsterReich (2017), Unbegleitete minderjährige Flüchtlinge (UMF) in Österreich. AsylKOORDINATEN. Infoblatt der Asylkoordination Österreich Nr. 1. Wien, asylkoordination österreich. Stand Februar 2017. 
BMASK - Bundesministerium für Arbeit, Soziales und Konsumentenschutz, Österreich (2016), Freiwilligenengagement. Bevölkerungsbefragung 2016. Studienbericht. Wien, Institut für Empirische Sozialforschung GmbH (IFES).

BMFSFJ - Bundesministerium für Familie, Senioren, Frauen und Jugend, Deutschland (2017), Engagement in der Flüchtlingshilfe. Ergebnisbericht einer Untersuchung des Instituts für Demoskopie Allensbach. Berlin.

Bourdieu P. (1983), Ökonomisches Kapital, kulturelles Kapital, soziales Kapital. In: KRECKEL R. (ed.), Soziale Ungleichheiten (Soziale Welt, Sonderband 2). Göttingen, Otto Schwartz \& Co. Verlag, pp. 183-198.

Han-Broich M. (2012), Ehrenamt und Integration. Die Bedeutung sozialen Engagements in der (Flüchtlings-)Sozialarbeit. Wiesbaden, Springer VS.

IOM - IntERnAtionale OrganisAtion Für Migration (2014), Die Gestaltung der Grundversorgung in Österreich. Wien, Mandelbaum Verlag.

Samochowiec J., Thalmann L. \& Müller A. (2018), Die neuen Freiwilligen - Die Zukunft zivilgesellschaftlicher Partizipation. Zürich, Gottlieb Duttweiler Institut.

ScHeIn E.H. (2009), Help. How to Offer, Give and Receive Help. San Francisco, Berrett-Koehler Publishers.

Schmidbauer W. (1977), Die hilflosen Helfer. Über die seelische Problematik der helfenden Berufe. Berlin, Rowohlt Verlag Reinbek.

Schweinschwaller T. (2018), Über das Helfen. Auswirkungen unterschiedlicher Logiken von freiwilligen und ehrenamtlichen MitarbeiterInnen am Beispiel der Flüchtlingshilfe. In: Krause S., Proyer M. \& Koenig O. (eds.), Gesellschaften/Welten/Selbst im [Um]Bruch. Wien, Wissenschaftliche Open Source Publikation, pp. 163-187.

SimSa R. (Projektleitung) et al. (2016), Beiträge der Zivilgesellschaft zur Bewältigung der Flüchtlingskrise - Leistungen und Lernchancen. Wien, Eigenverlag Wirtschaftsuniversität.

Zusätzlich wurden Auszüge aus persönlichen Gesprächen zwischen der Autorin und Freiwilligen für diesen Artikel herangezogen. 